- 56 hospital admissions were avoided

- 53 were referred on to the hospice Community Clinical Nurse Specialist Team

- two were admitted to hospital

- Only three people needed personal care.

Conclusion The project supported people to die where they wished to be cared for. It prevented a number of hospital admissions due to the rapid and skilled support of trained staff. The care component was not needed as the present provider was able to support within an appropriate timeframe. Feedback from families and carers was overwhelmingly positive.

\section{P-196 MOTOR NEURONE DISEASE: SUPPORTING PEOPLE TO DIE AT HOME IN LANCASHIRE AND SOUTH CUMBRIA}

1,2Simon Edgecombe, ${ }^{1,2}$ Sue Muller. ${ }^{1} S t$ John's Hospice, Lancaster, UK; ${ }^{2}$ Motor Neurone Disease Association, Lancashire and Cumbria, UK

\subsection{6/bmispcare-2017-hospice.221}

In 2016, twenty people with Motor Neurone Disease (MND) in the Lancashire and South Cumbria region were admitted and died in hospital. Following a meeting in early 2016 with the MND Association, the local hospices were approached to consider how the MND ice bucket challenge money could be utilised for future MND patient support. From this, local MND Association staff met with St John's Hospice staff to look at how the hospice could support MND patients through our already existing services.

The group looked at the gaps in services and staff knowledge, what causes a crisis leading to hospital admission, and what skills are required to keep people with MND at home, if that is their preferred place of care and death. The main issues often leading to admission were documenting and managing advance care planning; supporting cognitive impairment difficulties; and supporting family carers using practical skills such as taking blood gases, managing non-invasive ventilation (NIV) and using cough assist devices.

For family support and education, the day services team set up a 'STAR' group (Support, Time-out, Advice, Recovery: named by the attendees) for people with MND and their main carer. This addressed advance care planning; hospice services and what they can provide; support to carers; nutrition, and speech and language advice. This would run weekly, following on from the quarterly MND clinics, for four weeks. The team also set up a successful MND study day for professionals, which was well evaluated and covered diagnosis, prognosis, and symptom management. The Hospice at Home and ward teams are presently being developed in the practical skills listed above, in order to support people to stay in their own homes when near end of life. An update on this work will be included in the poster.

\section{P-197 REVIEW OF ST CHRISTOPHER'S HOSPICE 'NIGHT TIME' OUT OF HOURS SUPPORT FOR COMMUNITY}

Anne Nash. St Christopher's Hospice, London, UK

10.1136/bmjspcare-2017-hospice.222
Aim The aim of this 18 month service review was to reflect on the number of calls/contacts to the night co-coordinator triaging service were processing, to review on the number of emergency visits, their purpose, and if there was an increased usage of the medical on call support.

Background Since 11 January 2016, the emergency St Christopher's community team out of hours service has been supported by the inpatient nursing service. This is managed by the night coordinator triaging out of hours calls and if an emergency visit is required, (after $10 \mathrm{pm}$ to approximately 6.30-7 am), then a Band 6 night co-ordinator or Band 5 ward-based RN and HCA from the night team will visit the patient, using a wait and return cab service. There remains the on call support from the medical team. The St Christopher's community service supports 5 CCGs and, over night, four of the CCGs fund a community over- night district nursing service.

Methods For the first six months there was a small working group, (Medical Consultant, CNS Nurse Manager and Inpatient Matron), undertook an initial review of the service, with a focus of evaluating support from the medical team. This has continued with a three-monthly review by the Matron of the in-patient service. Each telephone contact from the patient or family member was recorded and any nurse visits by the inpatient nursing team. The out of hours visit was then reviewed retrospectively and themes analysed.

Findings and Conclusions On average per month there were 156 calls made to the night service triage service and eight nursing visits.

Of the 140 visits by the inpatient nursing team:

- 92 were primarily to administer medication for pain or end of life care

- 8 were for assistance with catheterisation issues

- 40 visits were for various other issues ranging from assessment of new symptoms, fixing $\mathrm{O} 2$ tubing, other hygiene issues and general nursing and repositioning issues.

All visits were assessed as appropriate use of this emergency support service, with the aim of supporting community patients at home and avoiding hospital admissions. The new service did not impact on medical on call support.

\section{P-198 I'M DYING - GET ME OUT OF HERE!}

Rachel Whiting, Helen Meehan, Wayne de Leeuw. Dorothy House Hospice Care, Wiltshire, UK

\subsection{6/bmjspcare-2017-hospice.223}

Background Responding to an identified need to reduce length of stay for patients at the end of life in hospital and funded through the Better Care Fund, our hospice, in partnership with the Specialist Palliative Care Team at the acute hospital, created an Enhanced Discharge Service to facilitate discharge to preferred place of care.

Aims

- Reduce inappropriate delay in discharge to preferred place of care at end of life

- Provide up to 24 hour care at home dependant on need

- Develop and implement a 'supportive care model' using end of life care stages of decline to support decision making.

Method We used a partnership approach working with the Local Authority, Clinical Commissioning Group and acute hospital. Appropriate patients were identified using a 'Stages of 
Decline' tool. Referrals were made by telephone to the Hospice Coordination Team. Band 3 carers were available to provide up to 24 hours care in patients' own homes dependant on need. There was ongoing review of care needs in liaison with the district nurse and hospice nurse specialists.

Results The service was effective and timely, supporting same or next day discharge. The service was responsive to patient and family need. The care provided was flexible, supporting patients in the last days of life alongside those awaiting a CHC Fast Track package of care. Good communication was fostered between the partner organisations. In one quarter 18 patients were discharged saving a total of 148 acute bed days. Conclusions The service has demonstrated a reduction in patient length of stay in the acute trust, improving patient flow in the wider health community. Funding has been secured for a further year and access will be extended to Discharge Liaison teams. Plans are now in place to address inequity across the patch and provide the service across other local authority areas.

\section{P-199 'EVERY DAY COUNTS' - PILOT OF A SERVICE FOR IMPROVING ACCESS AND CONSISTENCY IN PALLIATIVE CARE SEVEN DAYS A WEEK}

Christine Bloomer, Giovanna Cruz. Hospice Isle of Man, Douglas, Isle of Man, UK

10.1136/bmjspcare-2017-hospice.224

Background The National Institute for Health and Care Excellence (NICE) has highlighted the need for access to specialist palliative care seven days a week in order to improve the quality of care, experience and outcomes for patients and their families. (NICE, 2004; NICE, 2015) Little is known about the demand for a seven-day Palliative Care Clinical Nurse Specialist (PCCNS) service in a small island of $\sim 85000$ residents. Generalist services are available to patients on weekends and holidays.

Aim The aim of this pilot was to determine the need for, use of and the impact of a seven-day PCCNS service.

Method The pilot service was offered between 1 October 2016 and 31 March 2017 by the local hospice PCCNS team, the only specialist palliative care provider on the island. The service was established using the existing team of 5.2 full-time equivalent PCCNS, overseen by their manager. The PCCNS on duty for the weekend was available for urgent telephone calls and visits. For patients with anticipated need, contact was made as requested by PCCNS colleagues, and 'routine' work from the existing caseload was also undertaken. The service was publicised directly to existing patients, and health providers on the island. The number, source and reason for calls, visits and hospice or hospital admissions were recorded and analysed. Feedback was sought from patients, caregivers, the PCCNS team and health professionals.

Results The PCCNS team received 84 urgent calls, generating 59 visits and 113 additional calls. Main reasons for contact were for anxiety support (32\%), symptom management (28\%) and end of life care (16\%). Weekend service resulted in 12 in-patient admissions to hospice, $58 \%$ for end of life care.

Conclusion The number of contacts supports the case for weekend and bank holiday cover. A seven-day PCCNS service is feasible and beneficial to patients and their families.

\section{P-200 TRIAGE AND RAPID RESPONSE IN PALLIATIVE CARE: DOES IT MAKE A DIFFERENCE?}

Neil Grannon, Emer McKenna. Douglas Macmillan Hospice, Stoke-on-Trent, UK

\subsection{6/bmjspcare-2017-hospice.225}

Background There is national recognition that demand on palliative care services is undergoing rapid change. The shift in age demographics combined with increasing complexity requires a more adaptive approach to fulfilling the wishes of patients. NICE has previously suggested that as well as the traditional face to face visits during 9-5 that there should be telephone advice 24 hours a day. It goes on to say that face to face consultations outside of $9-5$, seven days a week is desirable. Consequently the community team has led an initiative to remodel the current service into one which 'Patients were seen at the right time, in the right place and by the right person.'

Aims

- Restructure the community team to enhance flexibility, deliver additional capacity, to be able to visit patients at any time of day or night

- The Community Team needs the flexibility to visit patients at any time of day or night

- Introduce triage for all new referrals to ascertain the actual patient need and the speed at which a response is needed

- Evaluate the change in outcomes.

Methods 1. Utilised change management and best practice tools to alter working patterns and responsiveness

2. A baseline review was conducted prior to implementation of triage. Post launch outcomes were reviewed results on a monthly basis to ensure relevant standards and targets had been adhered to.

Conclusion We have received positive feedback from staff and patients regarding the use and deployment of triage. Of the new referrals received in May 2017 we have identified 6\% of inappropriate referrals prior to first face to face meeting. In $3 \%$ of cases avoided inappropriate admissions to hospital and delivered an urgent response to $21 \%$ who previously may not have received a visit for 6.4 days

\section{P-201 THE HELPER SERVICE: VOLUNTEERS BRING COMPANIONSHIP AND A SENSE OF NORMALITY}

${ }^{1}$ Christine O'Sullivan, ${ }^{2}$ Debbie Hill. 'Marie Curie Hospice Liverpool, UK; ${ }^{2}$ Marie Curie, UK

10.1136/bmjspcare-2017-hospice.226

Background A scoping exercise undertaken by an end of life care charity revealed that people living with a terminal illness can be socially isolated, navigating the end of life journey can be complex and bewildering, carers can find the situation overwhelming and may long for respite. Many carers also feel isolated in bereavement.

Aim The Helper volunteer role was created in response to this feedback, with pilot services set up in 2009. Volunteers were trained to offer companionship and emotional support to people living with any terminal illness and their families, and to combat the issues listed above. 